\title{
Land Use and Impervious Surface Area Change by County in Pennsylvania (1985-2000) as Interpreted Quantitatively by Means of Satellite Imagery
}

\author{
Toby N. Carlson*
}

Pennsylvania State University, University Park, PA 16802, USA

\begin{abstract}
County averages of impervious surface area (ISA), and the urban and woodland fractions were determined for all 67 Pennsylvania counties from archived Landsat image data for the years 1985 and 2000. This paper focuses on changes in these quantities that occurred during the 15 -year period, the patterns of development across the state, and the possible reasons for the changes. Changes in ISA suggest a movement of population away from the largest cities into less populated areas where land is more abundant and where taxes and land values are lower. Most counties experienced at least some gain in ISA, primarily in low- and mid-level density development (mainly housing). Other than a few exceptions, Lackawanna and Erie Counties, counties experiencing dominant growth in the high density categories were generally less developed and with less impervious surface area than the average.
\end{abstract}

Keywords: Pennsylvania counties, impervious surface area, demographic changes, land use changes.

\section{INTRODUCTION}

Most counties and municipalities possess a considerable data base of land use and land use changes within their domains, which are customarily stored in a digital format accessable by a Geographic Information System (GIS). These data tend to be derived primarily from local surveys or aircraft imagery. Land use categories are identified, often imprecisely, with such designators as urban, residential, industrial, agricultural, etc. Definitions may be a bit arbitrary and possibly vary from municipality to municipality and from county to county, even within a particular state. For example, the category of agricultural use could imply a combination of farmland, pasture, land set aside to lie fallow or formerly agricultural fields earmarked for development. Woodland may refer to forested land but omit stands of trees on private property, in parks, on landfill, on strip mines or along highways.

Because of this proliferation of land use categories, analyses of image data from satellite or aircraft may be subdivided into a large number of categories. As successive analyses over the years of this type of land cover map tend to be created by different individuals, such maps might contain inconsistencies when two data sets some years apart are compared or when adjacent land areas are merged into a single map. Although lacking surface resolution and requiring subjectivity in supervised classification, digital satellite imagery does have the advantage of providing an essentially instantaneous view of an area the size of a county or larger over which a uniform methodology is imposed. Analyses based on satellite imagery, will have at least one advantage of being spatially and contextually consistent, especially if the images are processed by a single individual.

*Address correspondence to this author at the Pennsylvania State University, University Park, PA 16802, USA; Tel: 814-863-1582;

E-mail: tnc@meteo.psu.edu
Recent techniques have been developed which enable impervious surface area to be estimated from satellite imagery with some degree of confidence $[1,2]$. Impervious surface area (ISA) is defined as that surface which does not permit the vertical transfer of water or water vapor. Almost all impervious surface area visible by satellite consists of artificial building materials. As such, this parameter constitutes a highly useful and objective measure for assessing the human use of the land [3].

Impervious surface area fraction, fractional vegetation cover and five standard land use categories (urban, woodland, short vegetation, scrub or bare surface, water) are currently available, albeit at a slightly oversampled surface resolution $(25 \mathrm{~m})$ from Landsat imagery, for all of Pennsylvania for the years 1985 and 2000 [4]. These data constitute the basis for the present paper which will show by county in Pennsylvania the changes in three salient land use parameters: impervious surface area, fractional woodland and a category we define as 'open land' area during the 15year interval, 1985-2000.

We choose to focus on just these three parameters for several reasons: each sensitively reflects important uses of the land, are relatively easy to measure quantitatively and they exhibit considerable temporal consistency. Furthermore, fractional impervious surface area can tell us something about the density of building construction in each pixel [1], allowing us to show the dominant mode of land use change (whether as low-, medium- or high-density development), thereby providing a quantitative picture of how land use is changing.

\section{DATA SOURCES AND ANALYSIS METHODO- LOGY}

Impervious surface area, fractional vegetation cover, and five land use classes (urban, woodland, short vegetation, scrub/bare soil, and water) were determined by the author of 
this paper and an associate, Deborah Slawson, from Landsat TM image data for the years 1985, 1995 and 2000, the first and last of which are currently available on the PASDA web site. All TM images were georeferenced to a common UTM map base (spheroid Clarke 1866), datum NAD27, with a total root mean square error for geopositioning of less than one pixel. The nominal surface resolution of Landsat imagery is $30 \mathrm{~m}$. Each scene was slightly oversampled to a $25 \mathrm{~m}$ grid using the nearest neighbor criteria. Image classification was done using the ERDAS (1997) Imagine system [5].

Fractional vegetation cover and impervious surface area were calculated using methodology described by Carlson and Arthur [1,6]. Briefly, fractional vegetation cover is based on a mathematical transform of the normalized difference vegetation index (NDVI). Impervious surface area, which depends on the vegetation fraction, is calculated in those pixels classified as urban. As justified by Ridd [7], its numerical value is set equal to the fractional area in those urban pixels not covered by vegetation.

In constructing the original ISA and urban data, we made the assumption that pixels classified as urban in the year 1985 remained urban in 2000. Accordingly, we arbitrarily set those pixels (albeit a small minority of them) that were initially classified as urban in 1985 but not urban in 2000 to be urban in 2000. Originally, this expedient was necessary because it is unrealistic to expect that an impervious surface would be removed. Moreover, this particular PASDA data set was created initially to be used with the Clarke Urban Prediction model [8], which did not accept pixels which become de-urbanized. Since the error of image rectification was somewhat less than a pixel, some pixels were undoubtedly incorrectly located by a pixel or two. Thus, some pixels which appear to become de-urbanized might actually have remained urban, but were situated on the later (e.g., 2000) image on an adjacent location from their earlier (1985) location. The original urban pixel would still be counted as urban in the later image but so would the newly (and arbitrarily) created urban pixel in 2000. The result would be a somewhat inflated average ISA in the year 2000 and a somewhat larger increase ISA than actually occurred during the 15 -year interval. It is reasonable to suppose that changes in ISA of about $2 \%$ are within the error of estimate.

Averages of the various parameters were made for each county in Pennsylvania for the years 1985 and 2000. Fig. (1) shows the names and locations of the 67 counties.

\section{ANALYSES OF LAND USE CHANGE}

In order to show the amounts of impervious surface area, fractional woodland and fractional open land cover, including the changes which occurred during the 15-year interval, we have chosen to present the results in the type of representation shown in Figs. (2-6).

\section{ISA}

As of the year 2000, the highest values of ISA (Fig. 2) lie in the southeast, east and far western part of the state, i.e., counties including and surrounding the big cities of Philadelphia, Harrisburg, Scranton, Erie and Pittsburgh. Not surprisingly, these counties tend to be the most populous [9]. High values of ISA and ISA increases are found in some western counties, notably Allegheny County (which includes the city of Pittsburgh and which lost population) and two

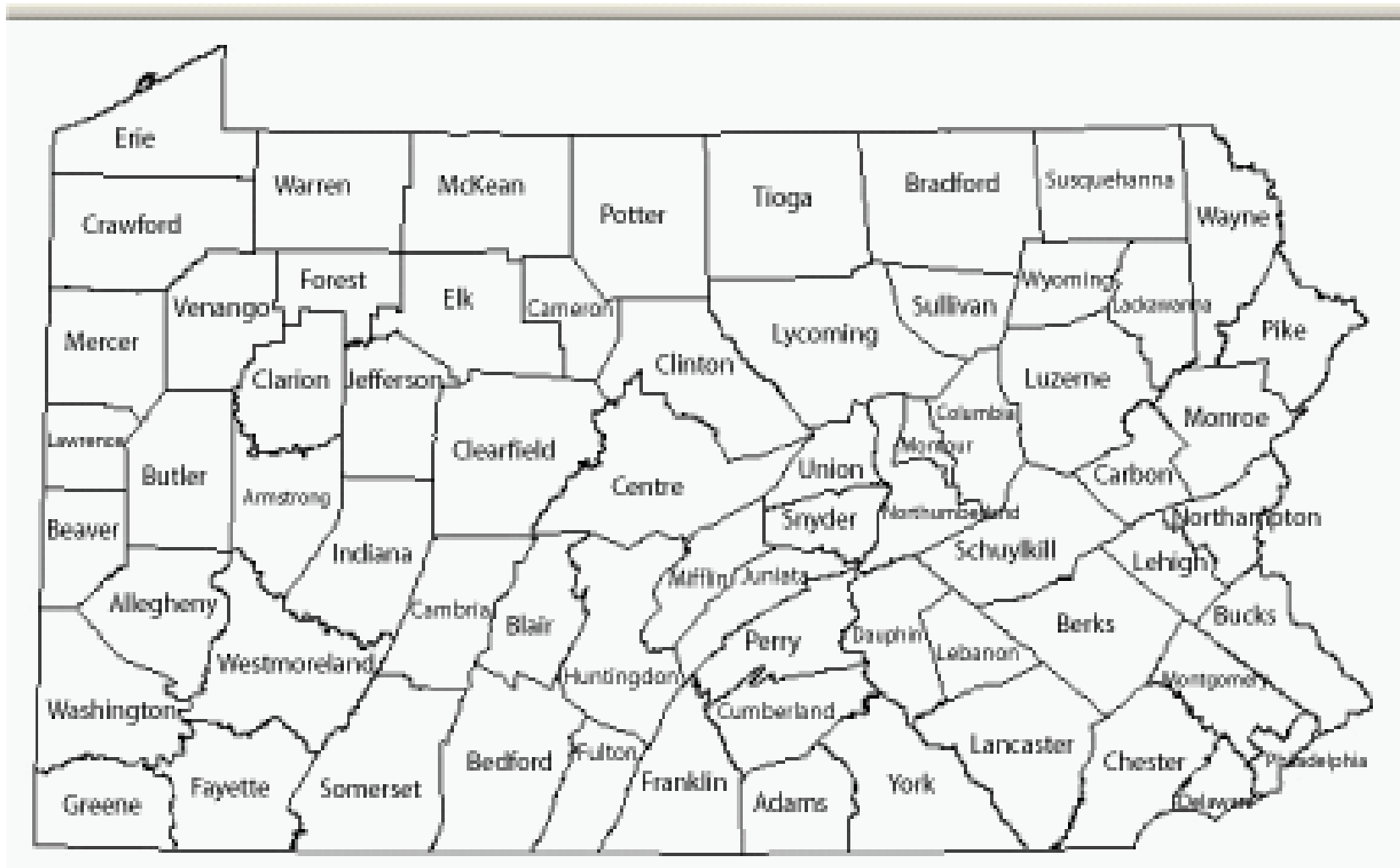

Fig. (1). County names in Pennsylvania. 


\section{Impervious surface area fraction (2000)}

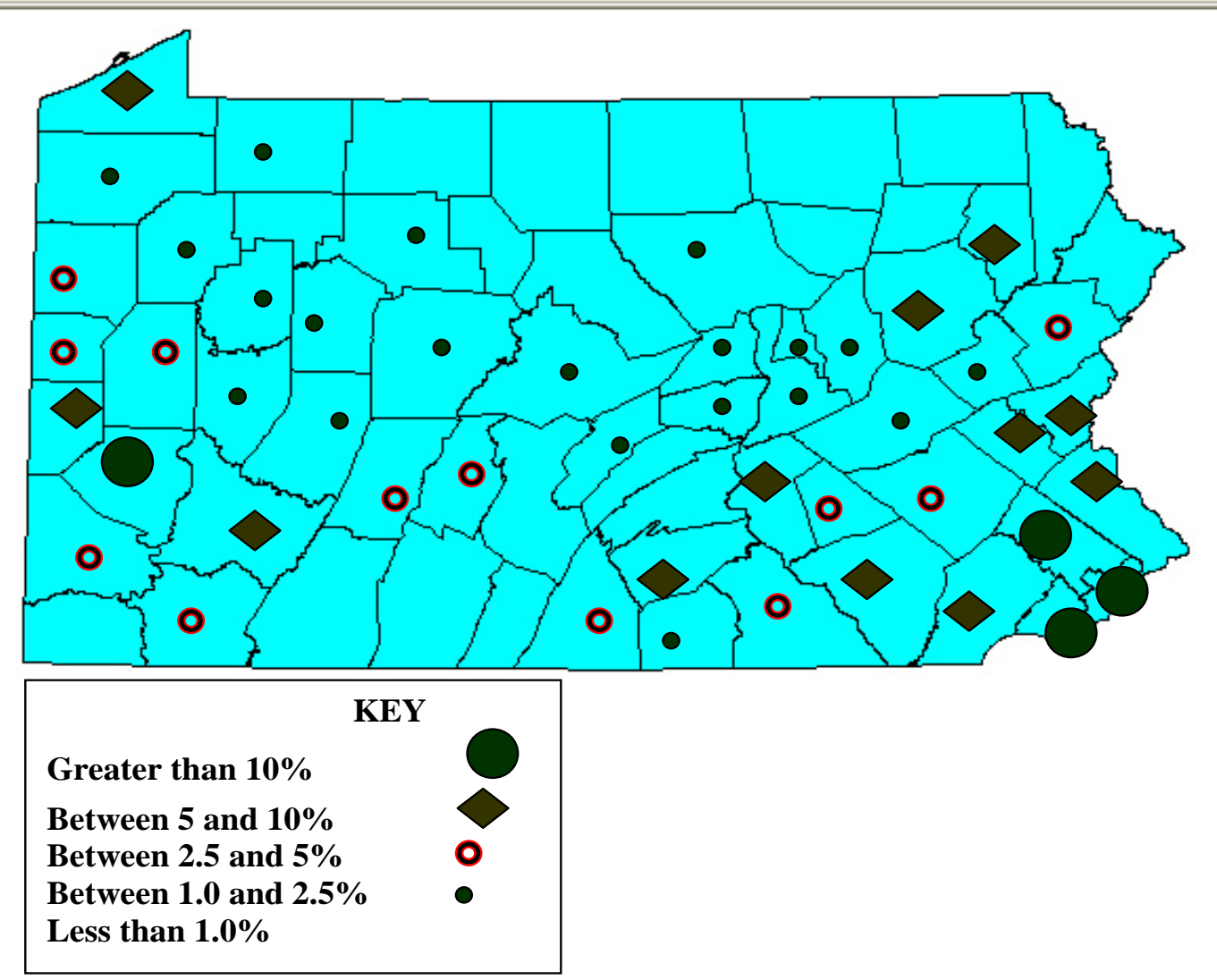

Fig. (2). Impervious surface area by county (year 2000).

adjacent counties, Westmoreland and Beaver.

Greatest increases in ISA (Fig. 3) are largely confined to areas already highly urbanized in 1985. Considerable increases in impervious surface area are found in these populous southeastern counties and in Cumberland and Dauphin counties, which encompass the capitol Harrisburg and its surroundings. Only small increases (less than $2 \%$ ) appear in most of the counties situated in the middle half of the state. Lackawanna County shows a startlingly large increase in ISA from an initially small value $(0.4 \%)$ in 1985 to $7.8 \%$ in 2000 . This county, which includes the city of Scranton, exhibited the most striking growth in ISA of any county in Pennsylvania, despite its having undergone some population loss. Indeed, several populous counties that experienced significant increases in ISA, Philadelphia and Allegheny Counties, experienced population losses, while Delaware County had almost no population increase. Dauphin and Columbia counties, however, do show significant increases in population in conjunction with significant increases in ISA [8]. Most counties, however, underwent no significant increase in ISA. These population changes [9] and their significance are discussed later in the paper.

\section{Woodland}

Woodland includes not just forests but any pixel classified as woodland - those pixels having trees as the dominant land cover. By this definition, nearly $60 \%$ of Pennsylvania is classified as wooded with the northern and central parts of the state having a woodland cover in excess of $65 \%$ (Fig. 4). Most of the counties exhibit little change in woodland area. Not surprisingly, the most urbanized areas exhibit the least amount of woodland. Although woodland cover over Pennsylvania has remained essentially constant over the past few decades and has actually increased from an historic low at the end of the $19^{\text {th }}$ century, a few counties did experience significant losses of woodland (more than $2 \%$ ), most notably Luzerne, Venango and Monroe counties. Lawrence, Allegheny, Erie, Lackawanna, Mercer and Delaware counties also experienced small but significant woodland losses. Largest losses were in the highly populated Allegheny County in the west and in the more suburban Delaware County in the southeast, which actually lost more than $15 \%$ of its woodland during the 15 -year interval.

\section{Open Land}

We define 'open land' as the sum of short vegetation and scrub/bare categories. In the present system, scrub/bare plus short vegetation categories would include almost all agricultural land use but also non-agricultural type of cover such as meadows and wetland. Agricultural land can be a rather nebulous category, including not just cropland but land lying fallow, bare surfaces on which plants have not yet emerged or have been harvested, and pasture. Conversely, the sum of the two classes which constitute our open land category serves both as an indirect index of agricultural land use and a direct measure of how much non-wooded land cover remains undeveloped. 


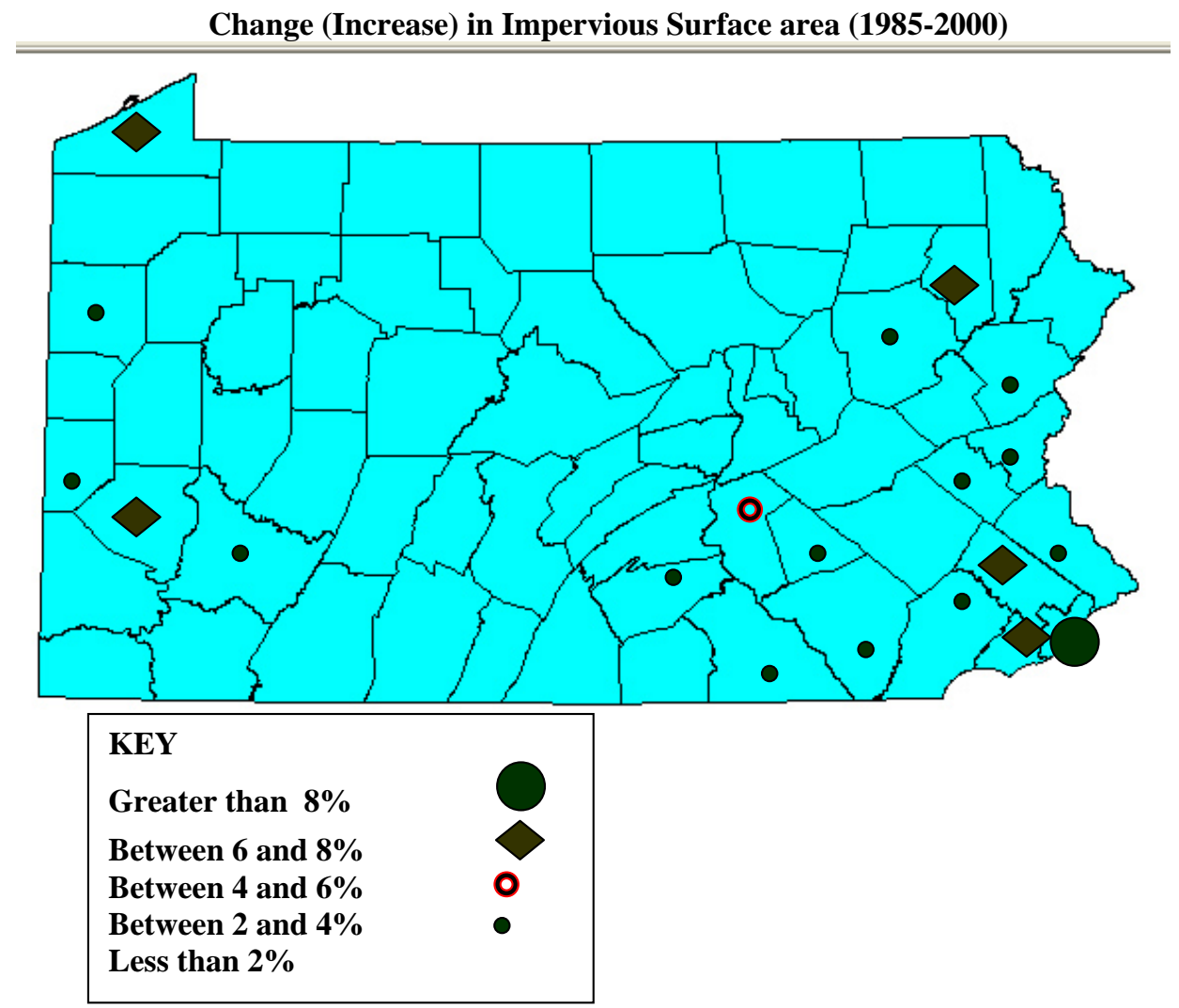

Fig. (3). Impervious surface area change by county during the 15-year period: 1985 - 2000.

\section{Percent woodland (2000)}

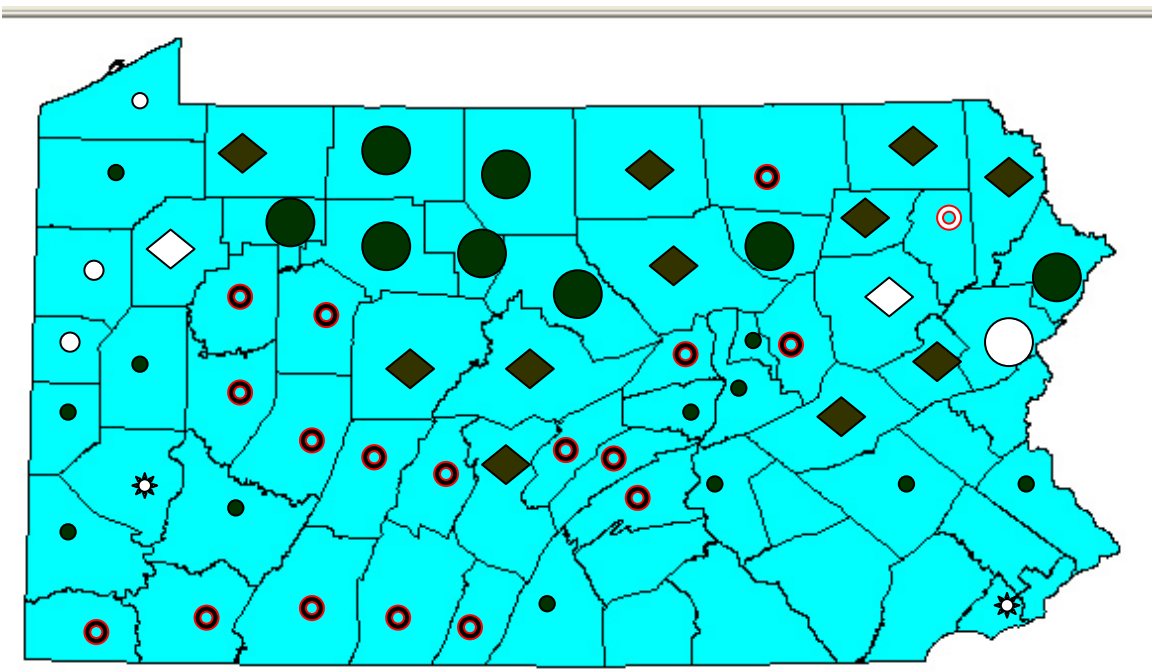

\begin{tabular}{|l|}
\hline KEY \\
Greater than $80 \%$ \\
Between 65 and $80 \%$ \\
Between 50 and $65 \%$ \\
Between 35 and $50 \%$ \\
Less than $35 \%$
\end{tabular}

Fig. (4). Percent Woodland Cover by county in the year 2000. Open symbols represent counties where woodland has decreased by more than $2.3 \%$ between 1985 and 2000. Starburst (enotes county with less than 35\% woodland in 2000 which has lost more than $6 \%$ of its woodland. 
Percent open land (2000)

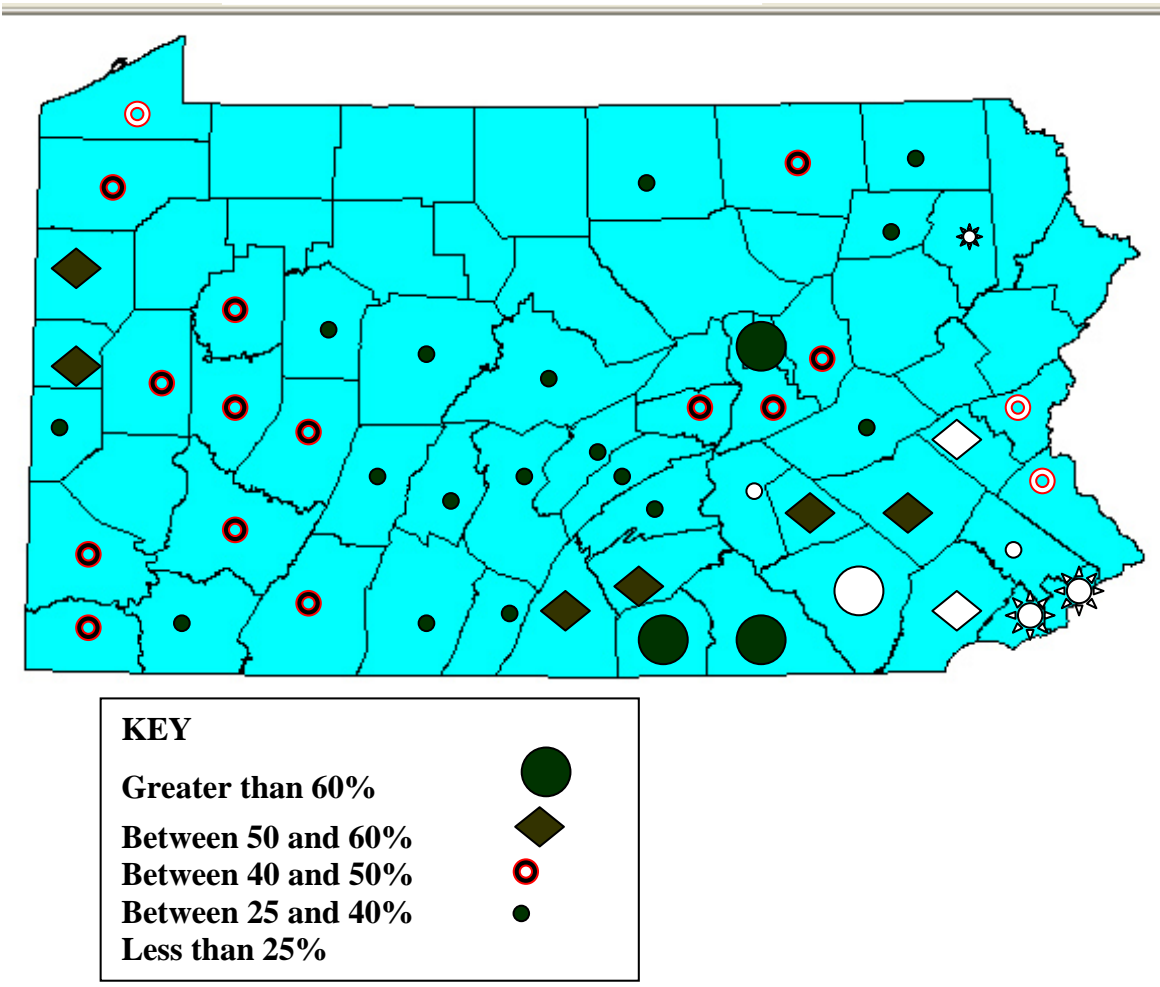

Fig. (5). Percent open land by county in the year 2000. Open symbols represent counties where open land has decreased by more than $5 \%$ between 1985 and 2000. Starburst (h) denotes county with less than 25\% open land in 2000 which has lost more than $5 \%$ (but less than $10 \%$ ) of its open land. Large starbursts represent counties with less than $25 \%$ open land which have lost more than $10 \%$ open land.

Fig. (5) is a map of open land cover. Large values are indicative of agricultural (farming) as a dominant land use category. Not surprisingly, counties with a large cover of woodland, which is not appropriate for farming, exhibit small amounts of open land, as do those highly populous counties with large amounts of impervious surface area. Significant losses of open land - largely the result of agricultural land losses - seem confined mainly to the southeastern part of the state, but also includes Lackawanna County. The exception to this regional bias is Erie County which, like Delaware County, also experienced significant losses in woodland (Fig. 4).

\section{Population and Density Changes}

Hebble et al. [1] showed that ISA pixels derived from 30 meter resolution Landsat imagery having values less than about 0.3 pertain to lot sizes greater than $2000-4000$ square feet; pixels having ISA values between 0.3 and 0.7 correspond to residential areas, particularly housing developments, and those with ISA values greater than about 0.7 correspond to industrial, commercial, highways or municipal structures (such as stores, schools, etc.) and sometimes to very dense housing developments. Accordingly, we have determined for each county the dominant ISA density category change (high, medium or low) between 1985 and 2000. Fig. (6) shows counties experiencing dominant changes in low density (L), medium density (M) and high density (H) categories. In a few cases two density categories show about equal changes, in which case both categories are indicated separated by a slash. For either the $\mathrm{L}$ or $\mathrm{M}$ category, but where sum of $\mathrm{L}+\mathrm{M}$ exceeds 0.8 (a high degree of residential development) or where the $\mathrm{H}$ category exceeded 0.5 (a high degree of commercial development), the symbols are slightly enlarged, underlined and printed in bold face. Bold-faced symbols therefore refer to counties in which either residential or commercial changes have been especially pronounced.

Fig. (7) is an ISA analysis of Uwchlan Township in Chester County, in which the population increased markedly (Fig. 10) in the low density category (generally less dense housing) (ref. Fig. 6), shown by the purple color. Housing developments are clearly easy to define by their row structure in which lines of houses are separated by parallel lines of small streets. Commercial, institutional and some dense housing developments, identified by bright red and orange pixels, favor locations along major roads, which appear in yellow. Black areas mostly contain open land. A photograph of a low-medium density housing unit in Chester County (purple areas in Fig. 7) is shown in Fig. (8).

Counties with primarily low density development are generally confined to the more populous eastern and western ends of Pennsylvania (Fig. 6). Medium density development seems more prevalent in the middle portion of the state. Almost all counties with primarily high density development are situated in clusters near the southwest and east central parts of Pennsylvania, although considerable high density housing development also took place in many of the populous eastern counties. An example of a high density housing development is shown in Fig. (9). This type of 


\section{Impervious surface density change analysis}

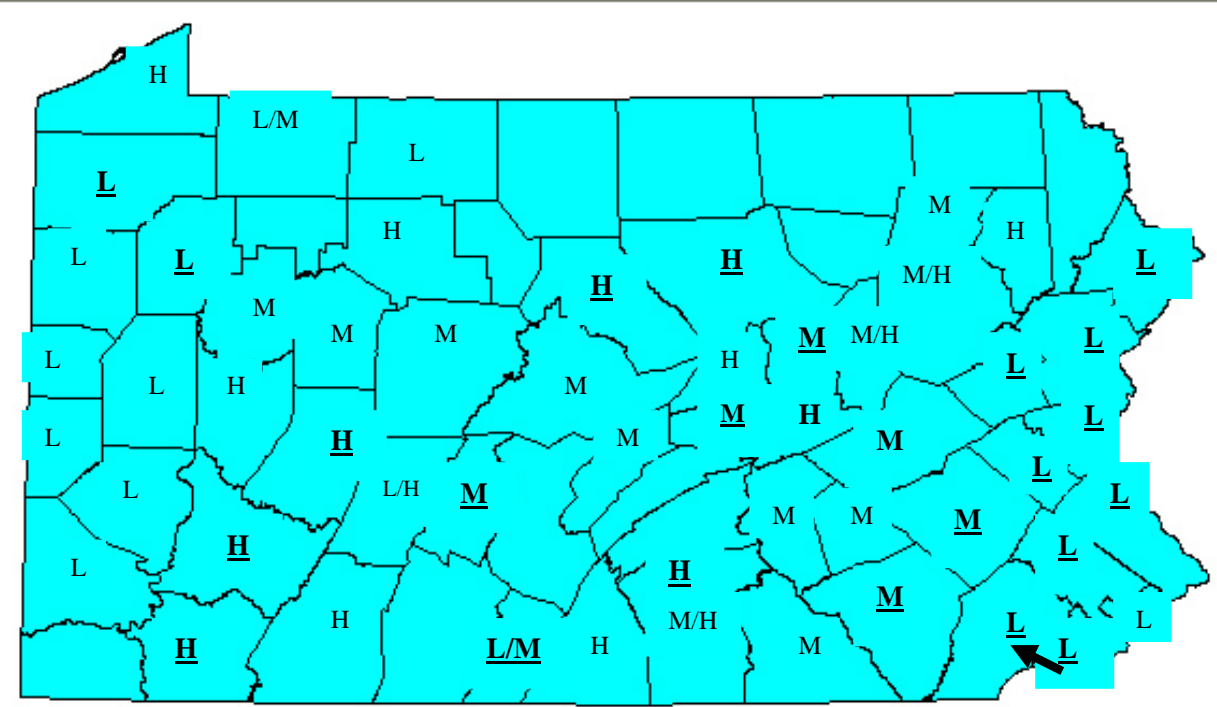

Fig. (6). Urban density change (1985-2000), based on the dominant impervious surface area (ISA) change by county. Symbols H, M and L refer, respectively, to the dominant class change being either in high density (ISA $>0.7$ ), medium density (ISA between 0.3 and 0.7 ) or low density (ISA < 0.3 ) categories. Underlined and slightly enlarged, bold-faced symbols refer either to counties in which the sum of the two residential categories $(\mathrm{L}+\mathrm{M})$ exceeds 0.8 or where the high density category $(\mathrm{H})$ exceeds 0.5 . Counties in which the ISA change was less than $0.5 \%$ are left blank. Counties in which changes in two categories are almost equal are represented by the appropriate symbols separated by a slash. The short arrow in the southeastern part of the state identifies Chester County (Fig. 7).

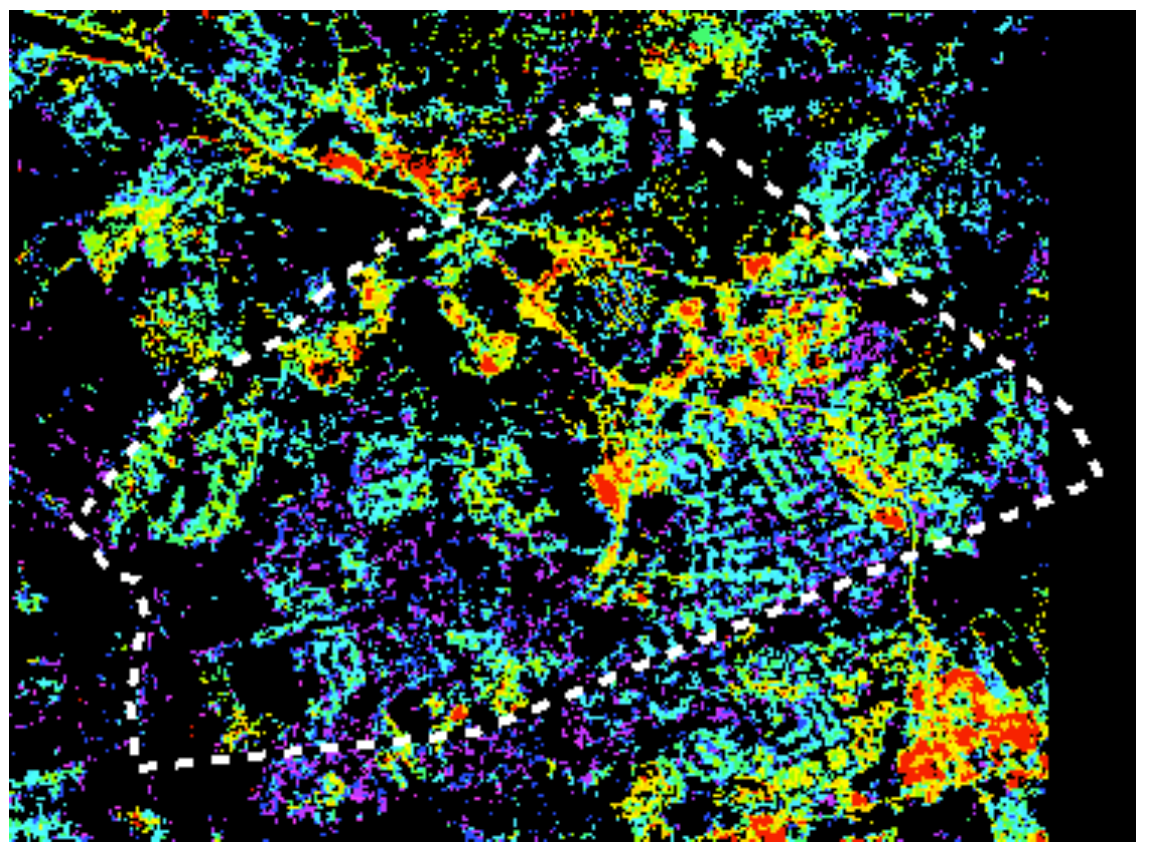

Fig. (7). ISA analysis for Uwchlan Township in Chester County, PA (short arrow in southeast in Fig. (6) denotes location of Chester County). Red, orange and yellow pixels consist of high density structures (H; ISA $>0.7)$, green and blue area are pixels representing medium density structures $(\mathrm{M} ; 0.3<\mathrm{ISA}<0.7)$ and purple and black are pixels of low density structures or open land (L: ISA $<0.3)$. The dashed border is the outline of the township.

development would appear as orange or red pixels in Fig. (7).

According to Olev Taremae [10], a planner for Northampton County, located on the eastern border of the state, the cluster of dominant low density changes along the eastern border of Pennsylvania, the Delaware River, is due to a flux of population from neighboring New Jersey and Delaware to Pennsylvania where the land values and taxes are lower. An exception is Philadelphia County, which experienced large increases in ISA and losses in population.

Philadelphia County illustrates a seemingly contradictory situation in which significant increases in ISA occurred in conjunction with the loss of population. Indeed, this type of correspondence is typical of the several highly populated areas, those encompassing the large cities of Philadelphia, Pittsburgh and Scranton-Wilkes-Barre. A reasonable 


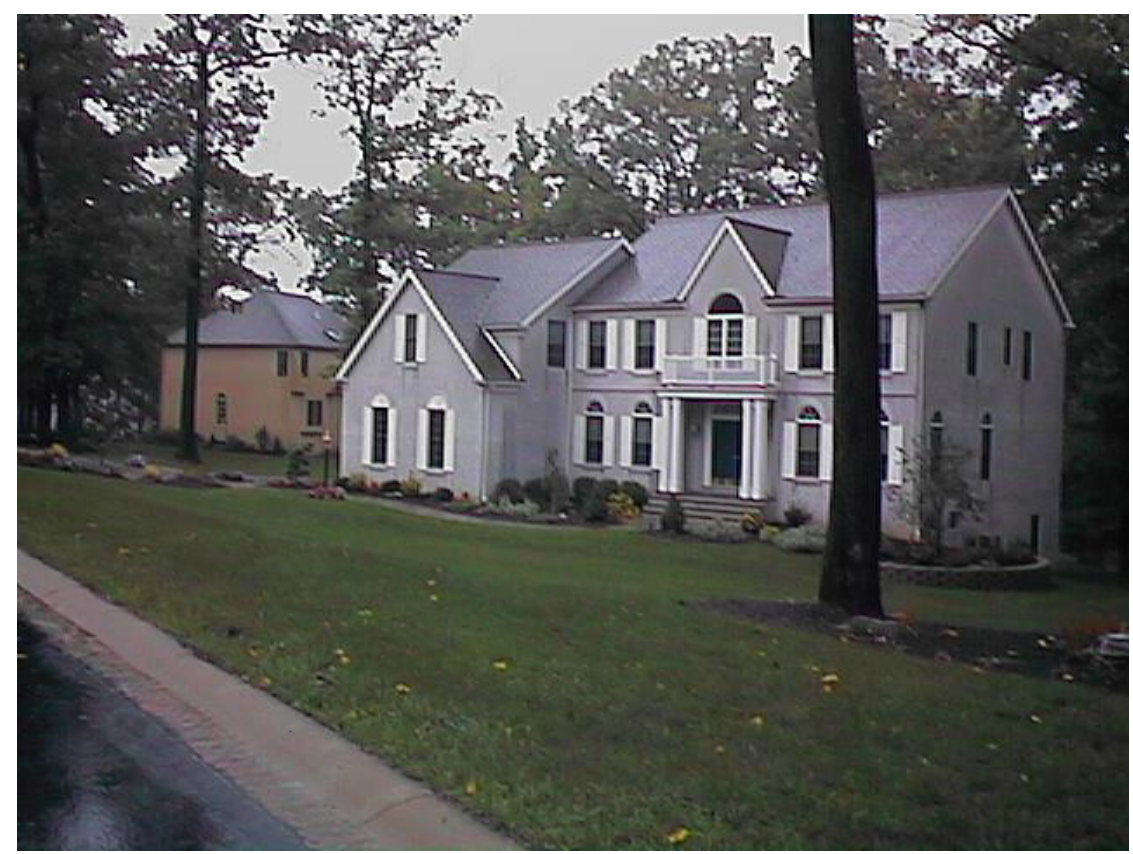

Fig. (8). Photograph of low density housing in Chester, County.

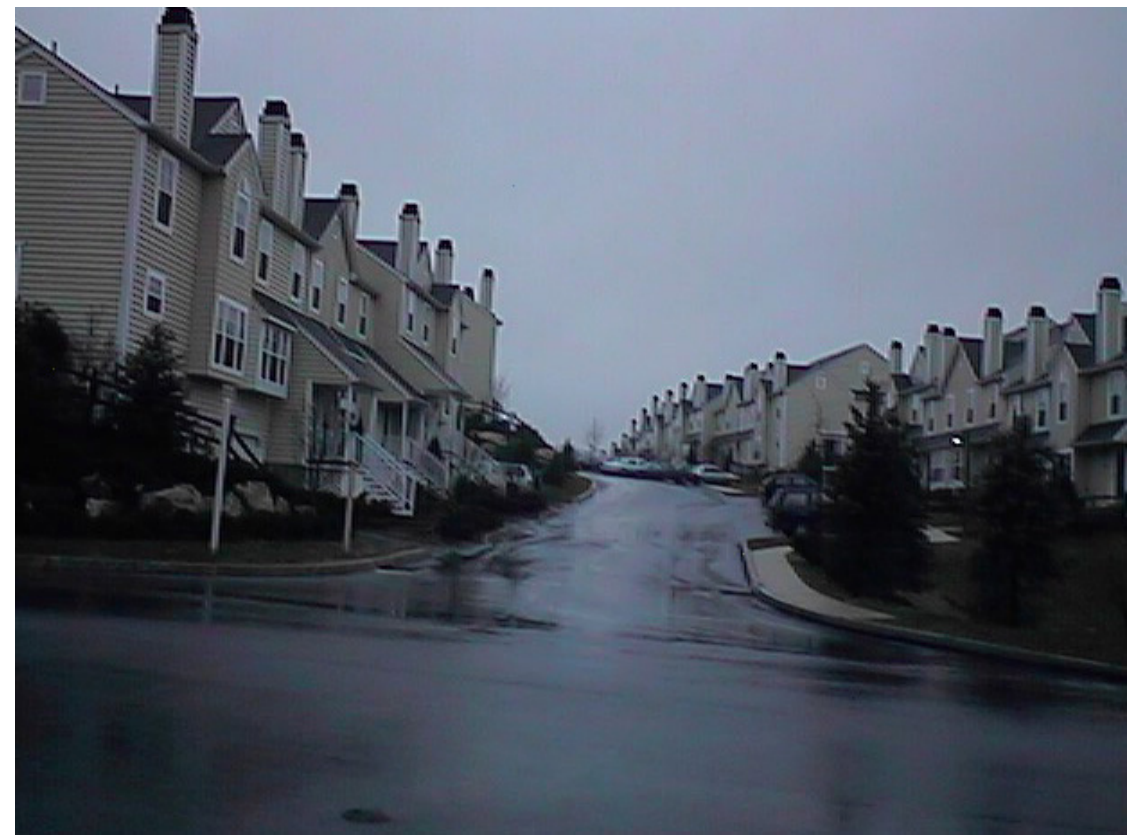

Fig. (9). Photograph of a high density housing development in Chester County.

explanation for this is that residents of the larger cities are moving out of congested environments and out of the county but also to exurbia where new shopping malls and industrial complexes are being built. Lackawanna County, in which Scranton is situated, is an illustration of this demographic movement. One exception is the capitol city of Harrisburg, situated in Dauphin County, in which both ISA and population significantly increased.

This influx of population to the eastern counties, reflected in Fig. (10) is occurring along the New Jersey and southern (eastern Maryland) borders. Numerous housing developments (reflected in the low density category of Fig. 6) were constructed in these border counties during the 1970s, 1980s and 1990s, which was followed by an influx of retail establishments and warehouses. A good example of this is Delaware County. One anomaly is Indiana County, which gained population during the 15-year period yet showed no significant increase in ISA.

Several counties experiencing strongly dominant growth in the high density category (greater than $50 \%$ of ISA change occurring in the high density category) were also counties in which very little impervious surface cover change occurred, such as Indiana and Fayette Counties in the southwestern part of the state, Erie and Lackawanna Counties being exceptions. In general those counties experiencing little change in ISA were also relatively unpopulated counties (compared to those with significant ISA increases) with less than $2 \%$ ISA cover. 


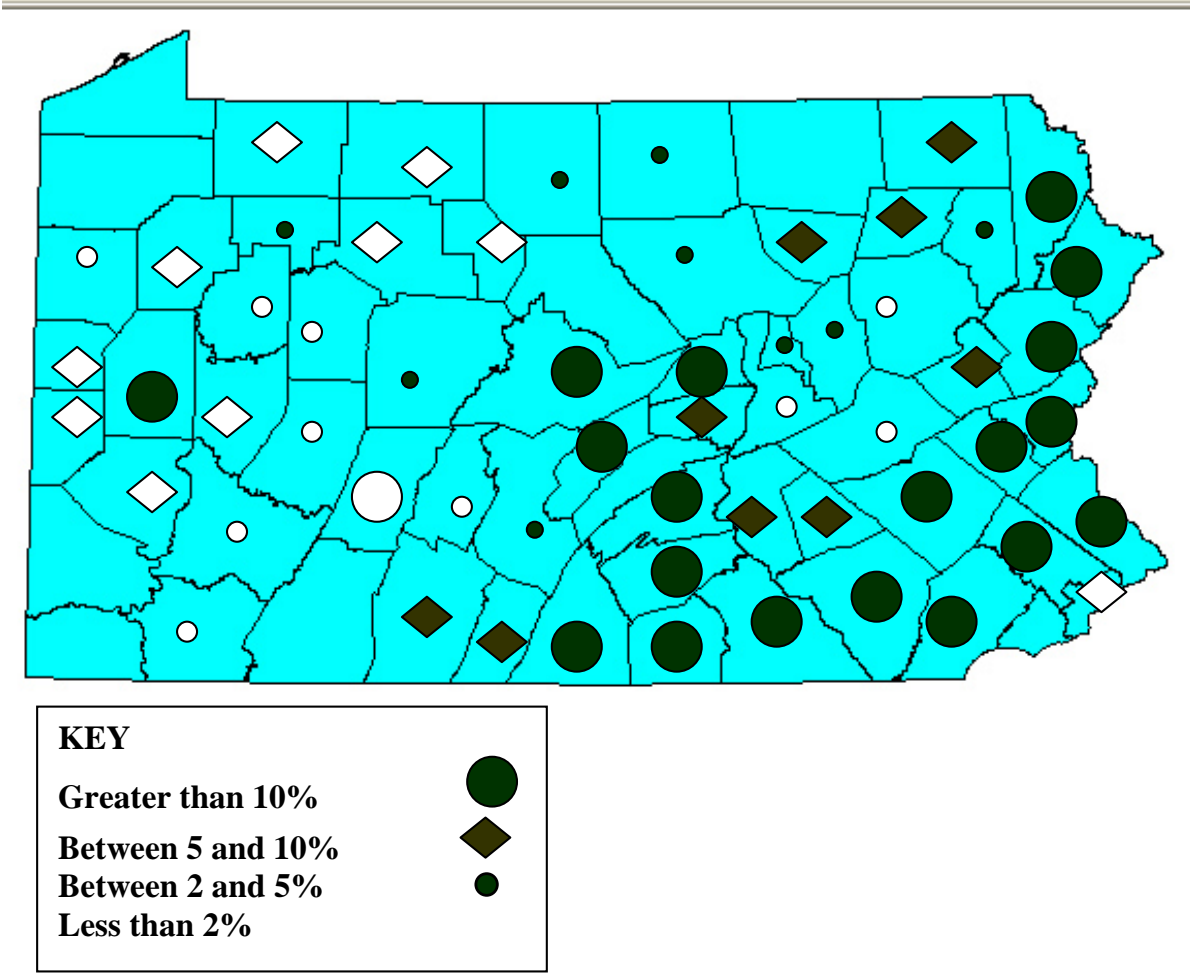

Fig. (10). Percent change in population by county from 1985 to 2000. Closed symbols represent counties whose population increased. Open symbols represent counties where population has decreased.

\section{SOME SPECIFIC EXAMPLES}

Table 1 summarizes land use change for a sampling of counties. These are now briefly discussed.

Mercer County, initially somewhat sparsely populated, experienced population losses but also underwent significant gains in developed land area and losses in woodland. Although the gains in development (or ISA) were primarily in the low density category, the county experienced some significant commercial development in the form or restaurants, Walmart stores, and industrial parks, such as Reynolds Aluminum. Residential development in the form of large housing developments also took place [11].
Venango County is an example of low population density which experienced little change in ISA. Venango did have some land development [12], as suggested by the loss in woodland, but otherwise this county had experienced population losses and some stagnation. Its growth, though small, was strongly in the lowest density category.

Lackawanna County, initially with a relatively low ISA $(0.4 \%)$ experienced considerable urbanization, which came at the expense of significant losses of open land and woodland (although more than $60 \%$ of the woodland remained in 2000). As such, Lackawanna's growth was among the largest of all the counties. A paradox, as discussed above, is that the county experienced population

Table 1. ISA, Urban, Woodland and Open Land Statistics for Selected Counties. Changes Refer to the Interval 1985 to 2000. Positive or Negative Symbols or the Number Zero After the Name Denote the Sign of Population Change

\begin{tabular}{|c|c|c|c|c|c|c|}
\hline County Name & $\begin{array}{l}\text { \%ISA } \\
(2000)\end{array}$ & $\begin{array}{l}\text { \% Urban } \\
\text { (2000) }\end{array}$ & $\begin{array}{l}\text { Change \% } \\
\text { Developed }\end{array}$ & $\begin{array}{l}\text { Change \% } \\
\text { Woodland }\end{array}$ & $\begin{array}{l}\text { Change \% Open } \\
\text { Land }\end{array}$ & $\begin{array}{l}\text { \% Woodland } \\
\text { (2000) }\end{array}$ \\
\hline Delaware (0) & 20.4 & 47.8 & 23.0 & -6.5 & -16.5 & 29 \\
\hline Allegheny (-) & 21.1 & 44.3 & 19.8 & -13.7 & -6.1 & 30 \\
\hline Erie (0) & 7.5 & 11.5 & 9.6 & -2.7 & -6.9 & 41 \\
\hline Dauphin (+) & 7.5 & 11.4 & 7.6 & -0.5 & -7.1 & 45 \\
\hline Lackawanna (-) & 7.8 & 12.5 & 10.3 & -3.6 & -6.7 & 64 \\
\hline Mercer (-) & 3.3 & 7.3 & 6.2 & $-2,4$ & -3.8 & 40 \\
\hline Venango (-) & 1.7 & 5.0 & 4.2 & -3.0 & -1.2 & 71 \\
\hline Pennsylvania (+) & 3.2 & 5.8 & 3.1 & -0.8 & -2.3 & 58 \\
\hline
\end{tabular}


losses, as discussed in the previous section. This growth in ISA is attributed to a number of industrial parks built during the 15-year period, along with other commercial developments and highways [13].

Dauphin County, encompassing Harrisburg and suburbs, gained population and experienced a significant increase in ISA while losing land to development, primarily at the expense of its open land (agriculture). During the 15 year interval, employment rose significantly along with the construction of thousands of new housing units [14].

Allegheny County is highly urbanized, reflecting the proximity of Pittsburgh and Philadelphia. Like Philadelphia County, in which the city of Philadelphia is situated, this county lost population while still experiencing significant increases in developed land area and ISA in the low density category. Delaware County also experienced a large increase in ISA but only a minimal population increase.

Northampton County, an example illustrating the influx of population in the eastern counties, gained population while undergoing large increases in developed land area at the expense of open land (agriculture). Northampton experienced moderate increase in ISA $(2-4 \%)$, primarily in the low-density category, the result of an urban spillover from Lehigh County (which also gained population) and from New Jersey [10], as mentioned in section 3

Erie's experienced a tremendous increase in ISA, (6-8\%). Erie's growth was accompanied by movement of population from the city of Erie to surrounding, less populated areas but without loss of population to the county. Residential growth was particularly strong amounting to almost $50 \%$ of the ISA change at the expense of farmland. Like many other parts of the state, this growth reflected the development of extensive suburbs, in this case around the city of Erie [15].

\section{CONCLUSIONS}

Pennsylvania as a whole underwent less than a $2 \%$ increase in impervious surface area (ISA), a $3 \%$ increase in urban land cover, and a small increase in population. Taken as a whole, Pennsylvania's urban growth seems to be characterized by movement of population away from the largest cities and from counties with more congestion, higher taxes and higher land prices, such as the neighboring state of New Jersey, to counties or areas of lower population density, lower taxes and lower land costs. This movement is characterized by a tendency for new residents of exurbia to create impervious surface on larger tracts of land, resulting in increased ISA and increased area classed as urban at the expense of open land and, to a lesser extent, woodland. Yet, in highly populated counties, even those which lost population, ISA often increased, sometimes quite dramatically, such as in Lackawanna, Philadelphia and Delaware Counties. This seeming paradox is explained by an exodus of population, not only out of the county but to the exurbs where shopping malls and other industrial sites were built.

Conversely, some highly rural counties such as Venango underwent population declines, probably because their economies would not adequately support new jobs other economic opportunities unless they happened to be the recipient of the population shifts from nearby urban centers.

\section{CONFLICTS OF INTEREST}

The authors confirm that this article content has no conflicts of interest.

\section{ACKNOWLEDGEMENT}

Declared none.

\section{REFERENCES}

[1] Hebble E, Carlson, TN, Daniel K. Impervious surface area and residential housing density: a satellite perspective. Geocarto Int 2001; 16: $13-8$.

[2] Elvidge CD. U. S. constructed area approaches the size of Ohio. EOS Bull 2004; 85: 233-4.

[3] Arnold ST, Gibbons CJ. Impervious surface coverage: the emergence of a key environmental indicator. J Am Plann Assoc 1996; 62: 241-58.

[4] Pennsylvania Spatial Data Analysis (PASDA). Available at: http:// www.pasda.psu.edu/

[5] ERDAS ERDAS IMAGINE, Tour Guide 8.3, ERDAS Corp 1997; pp. 454.

[6] Carlson TN, Arthur ST. The impact of land use - land cover changes due to urbanization on surface microclimate and hydrology: a satellite perspective. Glob Planet Change 2000; 25: 49-65.

[7] Ridd MK. Exploring a V-I-S (vegetation-impervious surface-soil) model for urban ecosystem analysis through remote sensing: comparative anatomy for cities. Int J Remote Sens 1995; 12: 216585.

[8] Clarke K, Gaydos L. Loose-coupling a cellular automaton model and GIS: long term urban growth predictions for San Francisco and Washington/Baltimore. Int J Geogr Inf Sci 1998; 12: 699-714.

[9] Pennsylvania Counties Population Data, 2002: Available from: http://www.recenter.tamu.edu/data/popc/popcs42.html.

[10] Olev Taremae, Lehigh Valley Planning Commission, 2007; Private communication, 2007 (Private communication)

[11] McDougall J, Mercer County Cooperative Extension.2007; (Private communication).

[12] Downs, J, Venango Planning Commission. 2007; (Private communication).

[13] Pitoniak S, Lackawanna Planning Commission. 2007; Private communication).

[14] Park J, Dauphin County Planning Commission. 2007: (Private communication).

[15] Elwell, M, Erie County Planning Commission 2007; (Private communication). 